\title{
Automated Posterior Cranial Fossa Volumetry by MRI: Applications to Chiari Malformation Type I
}

A.M. Bagci, S.H. Lee, N. Nagornaya, B.A. Green, and N. Alperin

\begin{abstract}
BACKGROUND AND PURPOSE: Quantification of PCF volume and the degree of PCF crowdedness were found beneficial for differential diagnosis of tonsillar herniation and prediction of surgical outcome in CMI. However, lack of automated methods limits the clinical use of PCF volumetry. An atlas-based method for automated PCF segmentation tailored for CMI is presented. The method performance is assessed in terms of accuracy and spatial overlap with manual segmentation. The degree of association between PCF volumes and the lengths of previously proposed linear landmarks is reported.
\end{abstract}

MATERIALS AND METHODS: Th-weighted volumetric MR imaging data with 1-mm isotropic resolution obtained with the use of a $3 T$ scanner from 14 patients with CMI and 3 healthy subjects were used for the study. Manually delineated PCF from 9 patients was used to establish a CMI-specific reference for an atlas-based automated PCF parcellation approach. Agreement between manual and automated segmentation of 5 different CMI datasets was verified by means of the $t$ test. Measurement reproducibility was established through the use of 2 repeated scans from 3 healthy subjects. Degree of linear association between PCF volume and 6 linear landmarks was determined by means of Pearson correlation.

RESULTS: PCF volumes measured by use of the automated method and with manual delineation were similar, $196.2 \pm 8.7 \mathrm{~mL}$ versus $196.9 \pm 11.0$ $\mathrm{mL}$, respectively. The mean relative difference of $-0.3 \pm 1.9 \%$ was not statistically significant. Low measurement variability, with a mean absolute percentage value of $0.6 \pm 0.2 \%$, was achieved. None of the PCF linear landmarks were significantly associated with PCF volume.

CONCLUSIONS: PCF and tissue content volumes can be reliably measured in patients with CMI by use of an atlas-based automated segmentation method.

ABBREVIATIONS: $\mathrm{CMI}=$ Chiari malformation type $\mathrm{I} ; \mathrm{PCF}=$ posterior cranial fossa

$\mathrm{T}$ he current radiologic definition of $\mathrm{CMI}$ is based on the degree of tonsillar herniation below the foramen magnum. However, imaging data with $\mathrm{x}$-ray, ${ }^{1} \mathrm{CT},{ }^{2}$ and $\mathrm{MR}^{3-7}$ gathered over the last several decades documented that $\mathrm{CMI}$ is also associated with a smaller than normal PCF. In most studies, length (1D $)^{5,6}$ and area $(2 \mathrm{D})^{8}$ measurements of certain PCF landmarks manually delineated on $\mathrm{x}$-ray film ${ }^{1}$ or on a midsagittal MR imaging ${ }^{6}$ were used for estimates of the PCF size. Volumetric (3D) assessment of the PCF, either with

Received October 8, 2012; accepted after revision October 11.

From the Departments of Radiology (A.M.B., S.H.L., N.N., N.A.) and Neurological Surgery (B.A.G.), University of Miami, Miami, Florida.

This work was supported by the National Institutes of Health (R01NS052122) and by a Small Grant Trainee Award from the Chiari \& Syringomyelia Foundation.

Paper previously presented in part at: 19th Annual Meeting of the ISMRM, Montreal, Quebec, Canada, May 7-13, 2011.

Please address correspondence to Noam Alperin, PhD, Department of Radiology (Locator: M869), Miller School of Medicine, University of Miami, 1150 NW 14th St, Suite 713, Miami, FL 33136; e-mail: NAlperin@med.miami.edu

-- Indicates open access to non-subscribers at www.ajnr.org

http://dx.doi.org/10.3174/ajnr.A3435
$\mathrm{CT}^{2}$ or MR imaging, ${ }^{4}$ further confirmed reduced PCF volume in patients with CMI compared with healthy controls. Through the use of manual delineation of the PCF and the brain tissue boundaries, Milhorat et $\mathrm{al}^{4}$ reported a smaller PCF volume as well as CSF volume, whereas the hindbrain volume was normal, leading to the notion of an overcrowded PCF. In a more recent study, they reported a small PCF volume only in "classic" CMI but not in "CMI mimicking" etiologies, thereby emphasizing the importance of the PCF volume for differential diagnosis of tonsillar herniation. ${ }^{7}$

Lirng et $\mathrm{al}^{9}$ used manual delineation of the PCF in MR imaging followed by image intensity-based segmentation of brain tissue and CSF to assess the effect of age and sex on the PCF volume and crowdedness in healthy subjects. They found that overall, men had a larger PCF and hindbrain volume, whereas women demonstrated a higher degree of crowdedness, which may explain the higher frequency of CMI in women.

Two other studies further suggest that the size of the PCF is also a strong predictor for surgical treatment outcome in CMI. Badie et $\mathrm{al}^{3}$ reported that a smaller ratio of the PCF volume relative to the supra- 
tentorial volume is associated with a better surgical outcome. A more recent study by Noudel et $\mathrm{al}^{10}$ used a semi-automated method to demonstrate that the response to the PCF decompression surgery is correlated with preoperation volume of PCF and the overall increase in the PCF volume after operation but not with the degree of tonsillar herniation or other tested morphologic measures. These limited data suggest that measurements of the PCF volume are likely to enhance both the diagnostic and prognostic reliability in CMI.

Despite the potential diagnostic and prognostic values of the PCF morphology, volumetric assessments of the PCF size are not commonly used in clinical practice because manual delineation of the PCF on multiple images is time-consuming. Manual length measurements of different landmarks of the PCF are less timeconsuming and are more commonly used as surrogate measures of the PCF volume. ${ }^{11}$ The most common 1D measurements are the lengths of the supraocciput and the clivus bones and the McRae and Twining lines at the midsagittal plane. ${ }^{4,6}$ However, these measurements are highly subjective and are strongly influenced by the MR imaging technique. Furthermore, it is not clear how well these linear (1D) measures correlate with the overall volume of the PCF. With the increasing evidence for the diagnostic potential of PCF measurement, there is a need for a robust automated method for reliable segmentation of the PCF. Moreover, because MR imaging is the primary technique used for diagnosis of CMI, it is important that such a method is available for MR imaging data.

A new approach for automated PCF parcellation is presented. The proposed parcellation uses atlas-guided segmentation, which has been successful in other cerebral regions. In addition to a measurement of the entire PCF volume, the method also provides measurements of the hindbrain tissue and CSF volumes. The robustness of the method is assessed by comparison with manual delineation in CMI data. Additionally, the degree of association between PCF volumes and linear landmarks is assessed.

\section{MATERIALS AND METHODS \\ Subjects}

PCF volumes were measured by use of MR imaging data from 3 healthy subjects who were scanned twice on 2 separate days (1 woman; age range, 29-36 years; mean age, $34 \pm 3$ years) and 5 symptomatic patients with CMI (3 women; age range, 23-48 years; mean age, $37 \pm 10$ years). MR imaging data from an additional 9 symptomatic patients with CMI ( 7 women; age range, 20-68 years; mean age, $37 \pm 15$ years) were used to create the CMI-specific atlas. All patients had cerebellar tonsillar herniation of at least $5 \mathrm{~mm}$ below the foramen magnum and presented with suboccipital headaches and numbness in the upper and/or lower extremities. Six patients had Valsalva-induced headaches. All subjects provided written informed consent, and the study was approved by the institutional review board.

\section{MR Image Acquisition}

The MR images used in the study were acquired with a 3T scanner (Magnetom Trio; Siemens, Erlangen, Germany). The structural analysis was performed on 3D T1-weighted image (magnetization prepared rapid acquisition of gradient echo) with the following

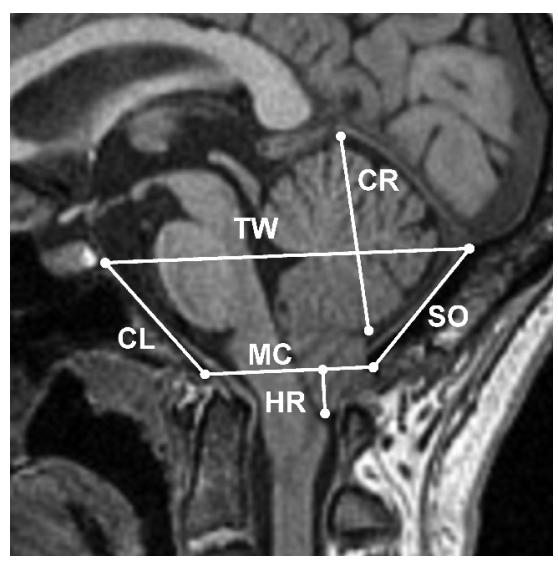

FIG 1. The 6 linear landmarks of the PCF superimposed on a midsagittal T1-weighted MR imaging from a patient with CMI: herniation (HR), McRae line (MC), clivus (CL), Twining line (TW), cerebellum (CR), and supraocciput (SO).

acquisition parameters: TR/TE/TI of 1900/2.89/900 ms, flip angle of $9^{\circ}$, FOV of $25.6 \times 25.6 \mathrm{~cm}$, and matrix size of $256 \times 256$, resulting in 1-mm isotropic resolution. Images were acquired in the sagittal orientation.

\section{Linear Measurements of the PCF}

Lengths of 6 midsagittal PCF structures were measured on the midsagittal T1-weighted image by a trained expert with 5 years of experience (S.H.L.). These structures were characterized as follows: 1) McRae line measured from the basion and the opisthion, 2) clivus length measured from the basion to the inferior boundary of the dorsum sellae, 3) Twining line measured from the dorsum sellae to the internal occipital protuberance, 4) height of the cerebellum, 5) supraocciput measured from internal occipital protuberance to opisthion, and 6) tonsillar herniation measured from the McRae line to tip of the cerebellar tonsil. An example of these markers overlaid on a midsagittal T1 MR image is shown in Fig 1.

\section{Manual Segmentation of PCF Volume}

The PCF was manually outlined on every sagittal section by use of the 3D Slicer software (http://www.slicer.org) by a single trained expert (A.M.B.) to avoid interobserver variability. Manual delineations were further reviewed and edited when needed by a neuroradiologist (N.N.). The PCF was anatomically bounded by tentorium cerebelli, occipital bone, clivus, and foramen magnum. The volume of the PCF was calculated by summation of the volume of each voxel within the manually created mask on each sagittal section.

\section{CMI-Specific PCF Atlas}

A PCF reference atlas, specific for CMI, was created from T1weighted images of 9 patients ( 7 women; age range, $20-68$ years; mean age, $37 \pm 15$ years). PCF labels were manually delineated on each subject image by an expert (A.M.B.) and were reviewed and confirmed for reliability by a neuroradiologist (N.N.). The T1weighted images were then affine-registered to Montreal Neurological Institute 152 space $^{12}$ and averaged to create the atlas template. The delineated PCF region from each of the 9 subjects was 


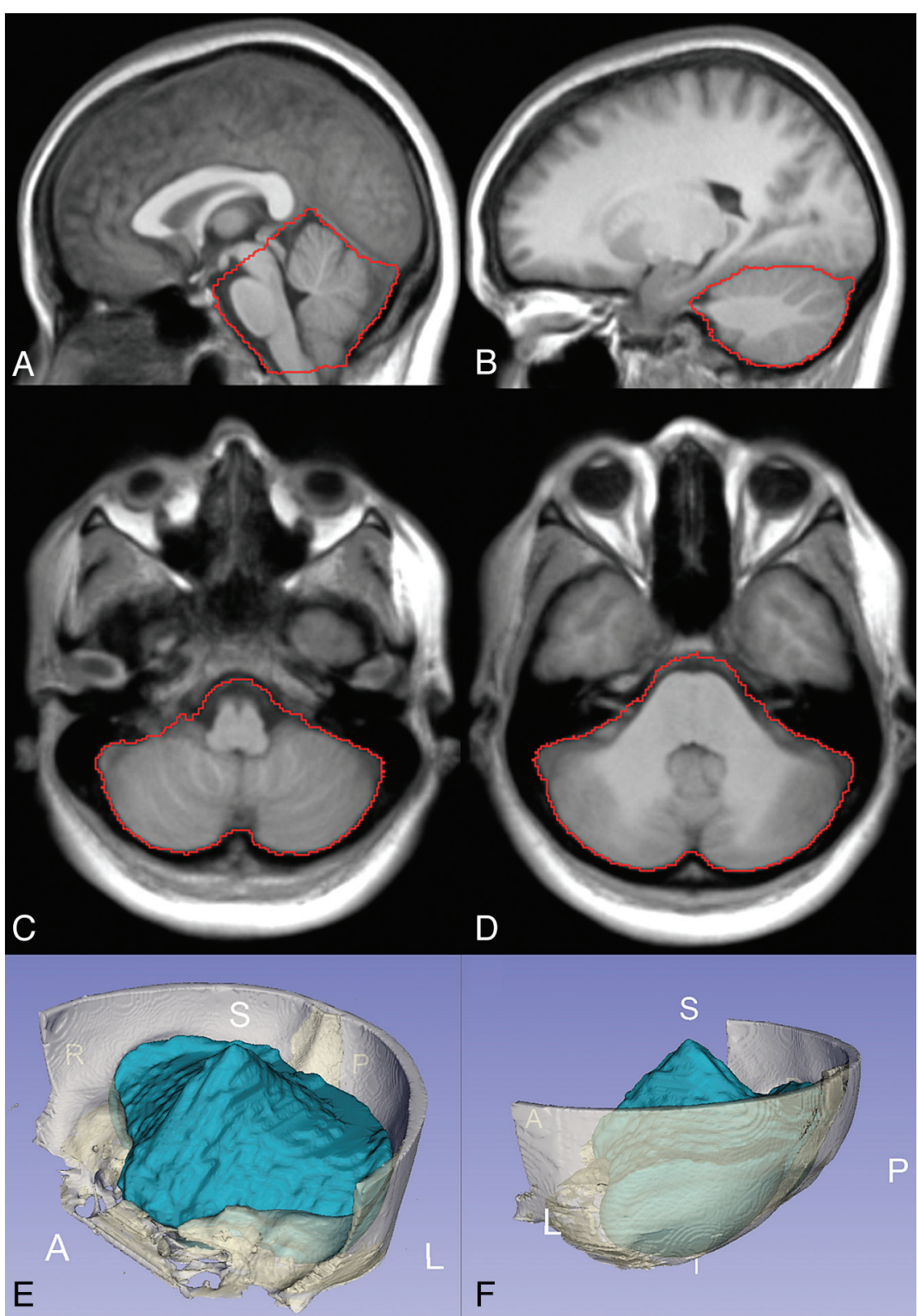

FIG 2. Chiari-specific atlas template and the boundary of the PCF compartment (red outline) shown in sagittal $(A$ and $B)$ and axial $(C$ and $D)$ planes. A 3D volumetric rendering of the PCF mask (blue) generated from the atlas template and surrounding cranium (white) are shown in $E$ and $F$.

\section{Automated Segmentation of PCF Volume}

Automated segmentation of the PCF volume is achieved by use of the previously described CMI reference atlas. First, a global linear transformation is applied to register the atlas template to the subject dataset by use of the FLIRT tool from FSL software package (http:// www.fmrib.ox.ac.uk/fsl). ${ }^{13}$ Only the brain region is used for the global registration to avoid adverse effects of cranial and extracranial structures on registration. ${ }^{14}$ After the global linear transformation, a more precise local alignment with a nonlinear registration is achieved by use of the FMRIB Nonlinear Image Registration Tool (FNIRT) from FSL, ${ }^{15}$ which is based on minimizing a sum-ofsquares cost function by use of a Levenberg-Marquardt modification of the Gauss-Newton method. Finally, the PCF mask is mapped to the subject MR imaging through the inverse of the registration to automatically segment the PCF volume.

\section{Automated Segmentation of Brain Tissue}

The proposed PCF parcellation method quantifies the volumes of tissue content of the PCF, which include the brain stem, cerebellum, medulla, and pons. Each pixel inside the PCF mask is labeled as gray matter, white matter, or CSF, by use of an algorithm that is based on the hidden Markov random field model and expectation maximization. ${ }^{16}$ The cerebellar tonsils extending beyond the foramen magnum are excluded as the result of PCF masking. A flow chart of the FSL implementation of the process for PCF segmentation and measurement of the PCF tissue volume is shown in Fig 3.

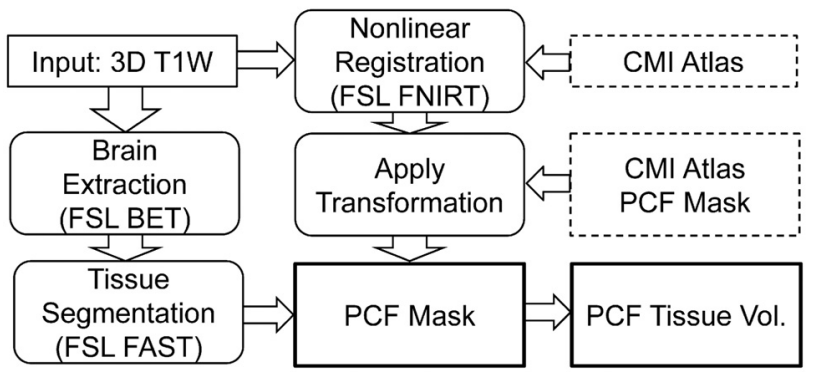

FIG 3. Flow chart illustrating the FSL implementation of the automated process for PCF segmentation and measurement of the PCF tissue volume.

then projected to the atlas space to generate an averaged PCF mask superimposed on the atlas space. The average reference atlas and the superimposed PCF regions are shown in Fig 2.
The intensity-based segmentation of the PCF tissues was compared with FreeSurfer-based (http://surfer.nmr.mgh.harvard.edu) segmentation of the hindbrain. The details of the FreeSurfer segmentation method are provided by Fischl et al. ${ }^{17}$ FreeSurfer segmentation is an atlas-based segmentation method, which used both the intensity distribution and the spatial relationships of previously defined brain regions. Labeled brain regions in the brain stem and gray and white matter in left and right cerebellum were combined and used as the hindbrain volume in this study.

\section{Assessment of Automated Segmentation Accuracy and Reliability}

The accuracy of the automated segmentation was assessed by comparing the automatically segmented PCF with manual seg- 

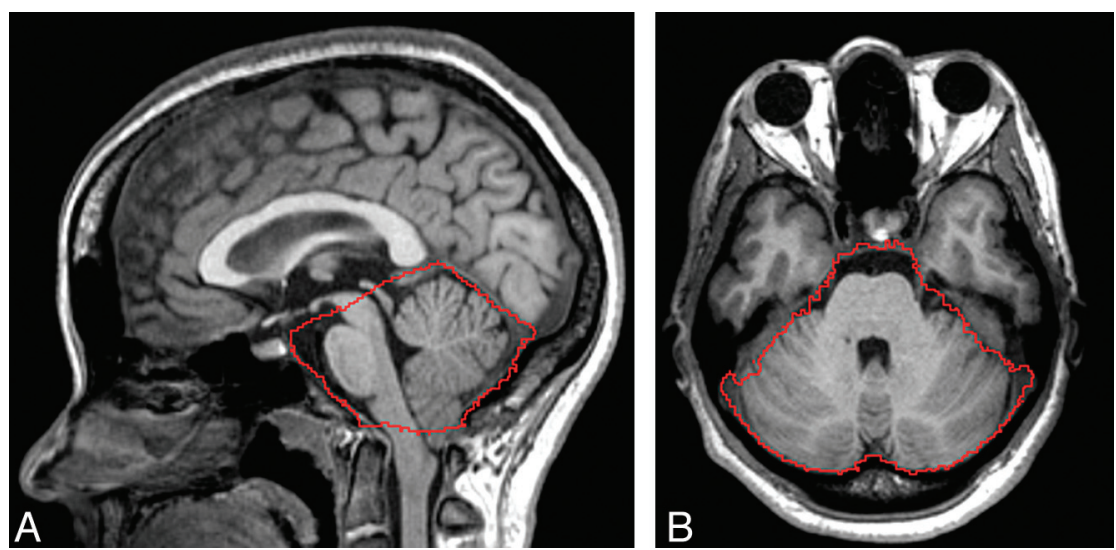

FIG 4. Outline of the PCF mask (red) generated by use of the proposed method on midsagittal (A) and axial (B) planes of Tl-weighted MR imaging of a patient with CMI.

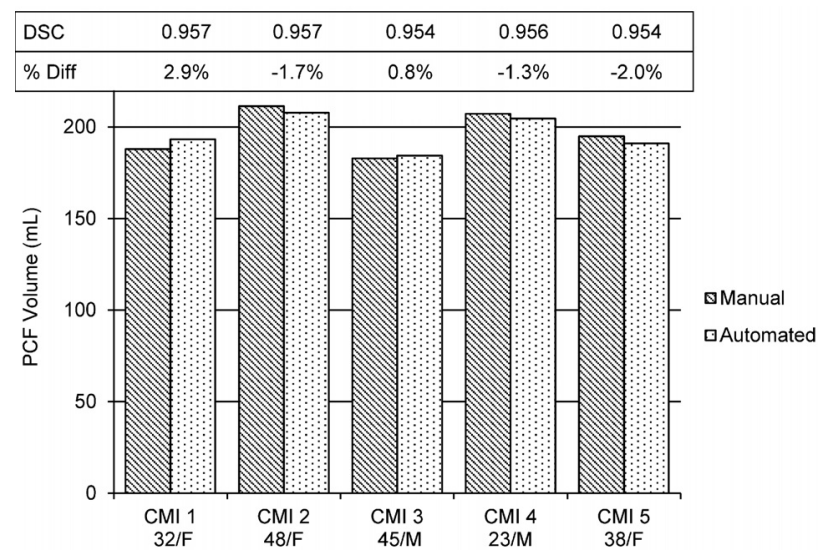

FIG 5. Comparison of PCF volume obtained manually and with the automated segmentation in CMI. The Dice similarity coefficient (DSC) and the relative percent change obtained for each of the 5 patients are shown above the volume bars.

Table 1: Repeated automated PCF volumes measurements

\begin{tabular}{ccccc}
\hline Subject & $\begin{array}{c}\text { Age/ } \\
\text { Sex }\end{array}$ & $\begin{array}{c}\text { PCF } \\
\text { Volume (mL), } \\
\text { Scan 1 }\end{array}$ & $\begin{array}{c}\text { PCF } \\
\text { Volume (mL), } \\
\text { Scan 2 }\end{array}$ & $\begin{array}{c}\text { Relative } \\
\text { Percentage } \\
\text { Difference }\end{array}$ \\
\hline 1 & $36 / \mathrm{M}$ & 186.1 & 186.8 & $0.4 \%$ \\
2 & $29 / \mathrm{M}$ & 207.5 & 205.9 & $0.8 \%$ \\
3 & $36 / \mathrm{F}$ & 194.7 & 195.6 & $0.5 \%$ \\
\hline
\end{tabular}

mentation in 5 patients with CMI. Percentage volume difference between the manual and the automated segmentations were calculated to test for systematic differences. Additionally, the Dice similarity coefficient was used to evaluate the degree of spatial overlap between the automatically and manually segmented PCF. The Dice similarity coefficient is defined as

$$
D S C=\frac{2^{\star} V(A \cap M)}{V(A)+V(M)}
$$

where $A$ and $M$ denote automated and manual segmentations, and $V$ denotes volume of the region. The value of Dice similarity coefficient ranges between $0-1$, representing no overlap to complete spatial overlap, respectively. A Dice similarity coefficient value $>0.7$ is considered as a good agreement between 2 compared measurements. ${ }^{18}$
The reliability of the automated segmentation across scan sessions was tested by means of MR imaging data from the 3 healthy subjects who were scanned twice on 2 separate days. The mean absolute percentage difference of the 2 measurements was calculated by dividing the absolute difference of the 2 measurements by their mean. A paired, 2 -tailed $t$ test was applied to determine the significance of differences between volume measurements obtained by use of 2 methods.

The posterior fossa brain tissue volume was measured in 5 patients with CMI by use of the proposed automated method and compared with measurements obtained by means of FreeSurfer. The mean percentage difference between 2 measurements and degree of spatial overlap was calculated. In addition, the derived crowdedness index, defined as the ratio of the tissue volume and the PCF compartment volume, were compared as well. Finally, linear association between PCF volumes and the length of the 6 different linear PCF markers were assessed by calculating the Pearson correlation coefficient and significance level by use of the data from the 14 patients with CMI. All statistical calculations were performed by use of MedCalc statistical software (MedCalc, Mariakerke, Belgium).

\section{RESULTS \\ PCF Volumes}

Examples of midsagittal and axial images with the identified PCF boundary and the 3D-rendered PCF volume from a representative patient with CMI are shown in Fig 4. The PCF volumes of each of the 5 patients with CMI measured with the 2 methods and the Dice coefficient representing the degree of overlap are shown in Fig 5. The mean and the SD of the manual and the automated segmentations of the PCF volumes in these 5 patients with CMI were similar $(197 \pm 11 \mathrm{~mL}$ and $196 \pm 9 \mathrm{~mL}$, respectively). The difference was not significant $(P=.7)$. The mean percentage volume difference was $-0.3 \pm 1.9 \%$. The mean degree of overlap (Dice coefficient) between the automatically and manually obtained PCF masks was $0.96 \pm 0.001$

The PCF volumes measured from the 3 healthy subjects who were scanned twice on 2 separate days and the relative percentage difference are listed in Table 1 . The mean absolute percentage difference was $0.6 \pm 0.2 \%$, with a range of $0.4 \%$ to $0.8 \%$; the difference between volume measurements was not statistically significant $(P=.995)$.

\section{PCF Crowdedness Indexes}

The posterior fossa brain tissue volume, crowdedness indexes of the 5 patients with CMI measured with the proposed method and with FreeSurfer, and the Dice coefficient are listed in Table 2. The mean and SD of the volumes measured with the proposed method and with FreeSurfer were $162 \pm 8 \mathrm{~mL}$ and $168 \pm 9 \mathrm{~mL}$, respectively. The tissue volumes measured by use of FreeSurfer were 
Table 2: Comparison of PCF tissue segmentation by means of the proposed method and FreeSurfer

\begin{tabular}{ccccccc}
\hline Patient & $\begin{array}{c}\text { PCF Tissue Volume } \\
(\mathbf{m L}) \text { (Proposed } \\
\text { Method) }\end{array}$ & $\begin{array}{c}\text { PCF Tissue Volume } \\
(\mathbf{m L}) \text { (FreeSurfer) }\end{array}$ & $\begin{array}{c}\text { Percentage } \\
\text { Volume Difference }\end{array}$ & $\begin{array}{c}\text { Dice } \\
\text { Coefficient }\end{array}$ & $\begin{array}{c}\text { Crowdedness } \\
\text { Index (Proposed } \\
\text { Method) }\end{array}$ & $\begin{array}{c}\text { Crowdedness } \\
\text { Index (FreeSurfer) }\end{array}$ \\
\hline 1 & 162.8 & 169.4 & $4.1 \%$ & 0.949 & 0.842 & 0.876 \\
2 & 172.1 & 181.6 & $5.5 \%$ & 0.939 & 0.828 & 0.874 \\
3 & 151.9 & 156.2 & $2.8 \%$ & 0.940 & 0.824 & 0.847 \\
4 & 170.1 & 174.0 & $2.3 \%$ & 0.948 & 0.831 & 0.850 \\
5 & 153.9 & 158.6 & $3.1 \%$ & 0.949 & 0.806 & 0.830 \\
\hline
\end{tabular}
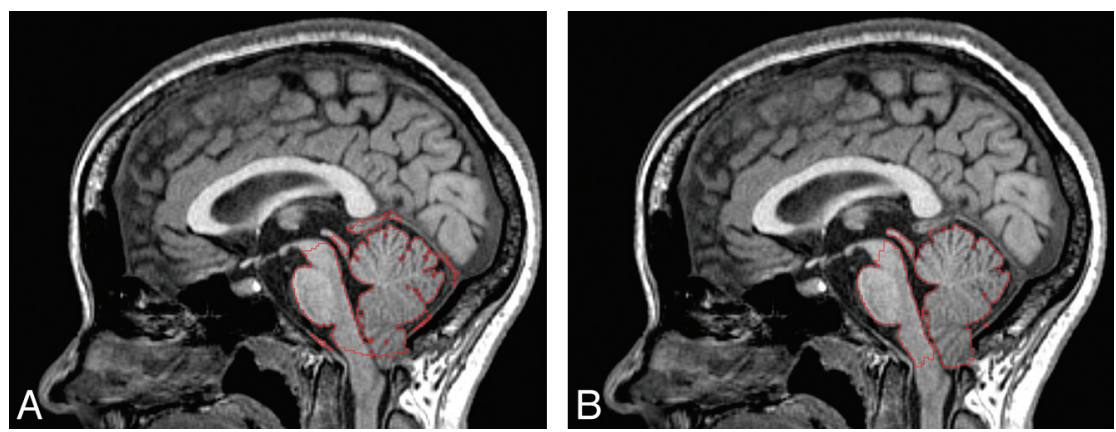

FIG 6. Outline of the PCF tissue masks (red) generated by use of the proposed method $(A)$ and FreeSurfer (B) in 1 of the patients with CMI.

consistently larger in each subject, with a mean percent difference of $3.6 \pm 1.1 \%(P=.005)$. The mean and SD of the spatial overlap were $0.945 \pm 0.004$. Images illustrating the tissue segmentation by the 2 methods are shown in Fig $6 A,-B$, respectively. The corresponding PCF crowdedness indexes were $0.826 \pm 0.012$ and $0.856 \pm 0.017$, respectively. Because FreeSurfer does not provide the PCF volume, the PCF volume obtained by the proposed method was used to estimate the PCF crowdedness obtained by use of the 2 methods.

None of the 6 linear PCF measures were significantly associated with the PCF volume. Five of the linear measures correlated positively with the PCF volume with the following corresponding Pearson correlation coefficients: supraocciput length $(r=0.38$, $P=.18)$, McRae line $(r=0.37, P=.20)$, clivus $(r=0.32, P=.27)$, Twining line $(r=0.30, P=.30)$, and length of cerebellum $(r=$ $0.29, P=.31$ ). As expected, the herniation length was negatively correlated with the PCF volume, with a Pearson correlation coefficient of $-0.17(P=.57)$.

\section{DISCUSSION}

Quantification of the PCF volume and the degree of PCF crowdedness were shown to be beneficial for differential diagnosis of tonsillar herniation ${ }^{7,11}$ and for prediction of surgical outcome. ${ }^{10}$ The lack of a reliable automated method for PCF volumetry by use of MR imaging, however, limits the clinical use of these PCF markers. Advanced automated methods for brain parcellation have matured in recent years and are becoming more widely used. ${ }^{16,17}$ This work represents adaptation of established brain segmentation techniques tailored toward PCF volumetry in CMI. The proposed atlas-guided PCF segmentation method is enhanced by the creation of a CMI-specific reference atlas that captures the altered PCF morphology associated with CMI. An excellent agreement between the proposed automated method and manual segmentation by an expert observer is evident by the small relative percentage difference of $-0.3 \pm 1.9 \%$ and the very high mean Dice coefficient of 0.96 . The delineation of the PCF obtained by use of the proposed automated method highly agrees with the manual delineation in terms of accuracy and spatial overlap in patients with CMI. Furthermore, a high degree of repeatability is evident from the small absolute percentage difference of $0.6 \pm 0.2 \%$ found by use of quantification of the repeated scans in 3 healthy subjects. The automated volume measurement of PCF is minimally affected by the normal variability in patient positioning in the MR imaging scanner.

The mean PCF volume measurement obtained in our small cohort of adult patients with CMI $(196 \pm 8.7 \mathrm{~mL})$ tends to be larger than previously reported CT and MR-based measurements of $186 \mathrm{~mL}$ by Nishikawa et al, ${ }^{11} 174 \pm 25 \mathrm{~mL}$ by Noudel et al, ${ }^{10}$ and $166 \pm 8 \mathrm{~mL}$ by Milhorat et al. ${ }^{7}$ The bias in the mean volume measurements may be attributed to the differences in the modalities and the possible differences in the segmentation protocols, particularly how the PCF boundaries were defined. Another contributing factor may be related to the difference in the sampling resolution of the volumetric data. In contrast to isotropic 1-mm $3 \mathrm{D}$ imaging used in this work, previous reports used 2D-based imaging with thicker sections for the volumetric measurements that can lead to measurement errors caused by large partial volume effect. In addition, the limited number of subjects used in this study to validate the proposed automated method against manual segmentation may not be representative of a CMI population in terms of PCF volume.

The tonsillar herniation in CMI has been attributed to overcrowding of the PCF as a result of a small PCF and normally developed brain tissue volume. ${ }^{7,10,11}$ Therefore, in addition to PCF volume measurement, accurate quantification of brain tissue volume is also critical. Our measurement of mean PCF tissue volume of $162.1 \pm 8.2$ also tends to be slightly larger than previously reported values of $156 \mathrm{~mL}$ by Nishikawa et $\mathrm{al}^{11}$ and $151.8 \pm$ $3.1 \mathrm{~mL}$ by Milhorat et al. ${ }^{7}$ However, the measurement of crowdedness, the ratio of PCF tissue volume to PCF volume of $0.826 \pm$ 0.012 , is in good agreement with the mean value of 0.833 reported by Nishikawa et al. ${ }^{11}$

The comparison of the hindbrain tissue volume measurements between the proposed method and FreeSurfer revealed a statistically significant mean difference of $3.6 \pm 1.1 \%(P=.005)$. The tissue volumes found through the use of FreeSurfer were consistently larger than volumes obtained by using the proposed method. As demonstrated in Fig 6, this difference is the result of 
the exclusion of the tonsillar tissue volume that descends below the foramen magnum. This tissue is excluded because it is outside the PCF and thus does not contribute to the PCF overcrowding.

Assessment of the associations between PCF volumes and the linear PCF markers revealed that none of the 6 measures were significantly associated with the PCF volume. The 5 linear landmarks of the PCF were all modestly positively correlated with the PCF volume. The lack of significance can be explained in part by the small sample. The length of herniation negatively correlated with the PCF volume, which is expected when a normal size cerebellum is compressed inside an increasingly smaller PCF.

The reference atlas used to guide the segmentation was prepared by use of MR images from patients with CMI, all of whom had tonsillar herniation $>5 \mathrm{~mm}$. Therefore, this atlas may not be optimal for segmentation of healthy subjects because of morphologic differences. However, for the purpose of reproducibility estimate, data from healthy subjects were used because repeated scans from patients with CMI were not available. Even with this limitation, an excellent reproducibility with an average difference of $0.6 \pm 0.2 \%$ is obtained, reflecting the robustness of the proposed method.

\section{CONCLUSIONS}

The PCF volume and the degree of crowdedness can be reliably quantified in MR imaging data of patients with CMI by use of an atlas-based approach. Automatically delineated PCF compartments were similar in volume and spatial overlap with those delineated manually by an expert observer. These early results suggest that automated segmentation could substitute for manual delineation of the PCF, thereby advancing the use of PCF parcellation for improved diagnosis and treatment decisions in CMI.

\section{ACKNOWLEDGMENTS}

The authors thank Lisa Kornse, BSN RN, for assistance with recruitment, Dr. Robert Quencer, MD, for supporting the Advanced Image Processing Lab, and the Evelyn F. McKnight Brain Institute for their support.

Disclosures: Ahmet Bagci—RELATED: Grant: Chiari \& Syringomyelia Foundation, *Comments: A \$10,000 Small Grant Award for Trainees. Noam Alperin—RELATED: Grant: NIH; *UNRELATED: Royalties: University of Illinois ( ${ }^{*}$ Money paid to institution).

\section{REFERENCES}

1. Nyland H, Krogness KG. Size of posterior fossa in Chiari type 1 malformation in adults. Acta Neurochir (Wien) 1978;40:233-42
2. Vega A, Quintana F, Berciano J. Basichondrocranium anomalies in adult Chiari type I malformation: a morphometric study. J Neurologic Sci 1990;99:137-45

3. Badie B, Mendoza D, Batzdorf U. Posterior fossa volume and response to suboccipital decompression in patients with Chiari I malformation. Neurosurgery 1995;37:214-18

4. Milhorat TH, Chou MW, Trinidad EM, et al. Chiari I malformation redefined: clinical and radiographic findings for 364 symptomatic patients. Neurosurgery 1999;44:1005-17

5. Karagoz F, Izgi N, Kapicioglu Sencer S. Morphometric measurements of the cranium in patients with Chiari type I malformation and comparison with the normal population. Acta Neurochir (Wien) 2002;144:165-71

6. Sekula RF Jr, Jannetta PJ, Casey KF, et al. Dimensions of the posterior fossa in patients symptomatic for Chiari I malformation but without cerebellar tonsillar descent. Cerebrospinal Fluid Res 2005;2:11

7. Milhorat TH, Nishikawa M, Kula RW, et al. Mechanisms of cerebellar tonsil herniation in patients with Chiari malformations as guide to clinical management. Acta Neurochir (Wien) 2010;152:1117-27

8. Krogness KG. Posterior fossa measurements, I: the normal size of the posterior fossa. Pediatr Radiol 1978;6:193-97

9. Lirng JF, Fuh JL, Chen YY, et al. Posterior cranial fossa crowdedness is related to age and sex: a magnetic resonance volumetric study. Acta Radiol 2005;46:737-42

10. Noudel R, Gomis P, Sotoares G, et al. Posterior fossa volume increase after surgery for Chiari malformation Type I: a quantitative assessment using magnetic resonance imaging and correlations with the treatment response. J Neurosurg 2011;115: 647-58

11. Nishikawa M, Sakamoto H, Hakuba A, et al. Pathogenesis of Chiari malformation: a morphometric study of the posterior cranial fossa. J Neurosurg 1997;86:40-47

12. Mazziotta J, Toga A, Evans A, et al. A probabilistic atlas and reference system for the human brain: International Consortium for Brain Mapping (ICBM). Philos Trans $R$ Soc Lond B Biol Sci 2001;356:1293-322

13. Jenkinson M, Smith S. A global optimisation method for robust affine registration of brain images. Medical Image Analysis 2001;5:143-56

14. Smith SM. Fast robust automated brain extraction. Hum Brain Mapp 2002;17:143-55

15. Andersson JL, Smith SM, Jenkinson M. FNIRT: FMRIB's non-linear image registration tool. Annual Meeting of the Organization Hum Brain Mapping. Melbourne, Australia, 2008

16. Zhang Y, Brady M, Smith S. Segmentation of brain MR images through a hidden Markov random field model and the expectation-maximization algorithm. IEEE Trans Medical Imaging 2001;20:45-57

17. Fischl B, Salat DH, Busa E, et al. Whole brain segmentation: automated labeling of neuroanatomical structures in the human brain. Neuron 2002;33:341-55

18. Cabezas M, Oliver A, Llado X, et al. A review of atlas-based segmentation for magnetic resonance brain images. Comput Methods Programs Biomed 2011;104:e158-e177 